\title{
Effectiveness of a lifestyle intervention in promoting the well-being of independently living older people: results of the Well Elderly 2 Randomised Controlled Trial
}

\author{
Florence Clark, ${ }^{1}$ Jeanne Jackson, ${ }^{1}$ Mike Carlson, ${ }^{1}$ Chih-Ping Chou, ${ }^{2}$ Barbara J Cherry, ${ }^{3}$ \\ Maryalice Jordan-Marsh, ${ }^{4}$ Bob G Knight, ${ }^{5}$ Deborah Mandel, ${ }^{1}$ Jeanine Blanchard, ${ }^{1}$ \\ Douglas A Granger, ${ }^{6}$ Rand R Wilcox, ${ }^{7}$ Mei Ying Lai, ${ }^{2}$ Brett White, ${ }^{8}$ Joel Hay, ${ }^{9}$ \\ Claudia Lam, ${ }^{2}$ Abbey Marterella, ${ }^{1}$ Stanley P Azen ${ }^{10}$
}

For numbered affiliations see end of article.

\section{Correspondence to Dr Florence Clark, Division of Occupational Science and Occupational Therapy, School of Dentistry, University of Southern California, 1540 Alcazar Street, CHP 133, Los Angeles, CA 90089-9003, USA; fclark@usc.edu}

Role of study sponsors: All data acquisition was inspected and reviewed annually by the designated data safety and monitoring board.

Accepted 28 April 2011 Published Online First 2 June 2011

\section{ABSTRACT}

Background Older people are at risk for health decline and loss of independence. Lifestyle interventions offer potential for reducing such negative outcomes. The aim of this study was to determine the effectiveness and cost-effectiveness of a preventive lifestyle-based occupational therapy intervention, administered in a variety of community-based sites, in improving mental and physical well-being and cognitive functioning in ethnically diverse older people.

Methods A randomised controlled trial was conducted comparing an occupational therapy intervention and a no-treatment control condition over a 6-month experimental phase. Participants included 460 men and women aged $60-95$ years (mean age $74.9 \pm 7.7$ years; $53 \%<\$ 12000$ annual income) recruited from 21 sites in the greater Los Angeles metropolitan area.

Results Intervention participants, relative to untreated controls, showed more favourable change scores on indices of bodily pain, vitality, social functioning, mental health, composite mental functioning, life satisfaction and depressive symptomatology (ps<0.05). The intervention group had a significantly greater increment in quality-adjusted life years $(p<0.02)$, which was achieved cost-effectively (US $\$ 41$ 218/UK £24 868 per unit). No intervention effect was found for cognitive functioning outcome measures.

Conclusions A lifestyle-oriented occupational therapy intervention has beneficial effects for ethnically diverse older people recruited from a wide array of community settings. Because the intervention is cost-effective and is applicable on a wide-scale basis, it has the potential to help reduce health decline and promote well-being in older people.

Trial Registration clinicaltrials.gov identifier: NCT0078634

\section{INTRODUCTION}

The expansion of the elderly population is likely to be accompanied by declines in physical health, mental well-being and functional ability. ${ }^{1-3}$ Fortunately, age-related declines can be delayed by engagement in a healthier lifestyle, ${ }^{2}{ }^{4}$ a result that highlights the need to develop interventions that promote modifiable healthy behaviours in older people.

In response to this need, in 1997, our investigative team completed the University of Southern
California Well Elderly study (Well Elderly 1), a randomised controlled trial of the efficacy and cost-effectiveness of a 9-month lifestyle intervention (now called Lifestyle Redesign ${ }^{\circledR}$ ) designed to slow age-related declines among independently living elders. ${ }^{6}$ In this study, which included 361 elders from two large federally subsidised housing complexes, a reliable positive intervention effect was obtained cost-effectively for a wide range of outcomes, such as life satisfaction, role functioning and self-rated physical and emotional health. ${ }^{6-8}$ Although additional trials have underscored the value of lifestyle interventions for older people, such research has typically been performed in a single setting only, has involved a relatively small sample size or lacked a cost-effectiveness evaluation. $^{9-11}$

This article reports on the University of Southern California Well Elderly 2 study, which assessed the Lifestyle Redesign intervention's effectiveness among ethnically diverse elders in communitybased settings, the majority of whom are from populations at risk for health disparities. In contrast to efficacy, which pertains to an intervention's success under favourable conditions that maximise the experimental effect, the effectiveness of an intervention refers to its performance under less tightly controlled but more realistic circumstances that characterise complex real-world settings. ${ }^{12}$ To assess effectiveness, we (1) sampled elders from 21 locations with wide variation in agency 'buy-in'; (2) included a 6-month intervention period to be responsive to real-world feasibility concerns and (3) sampled a high proportion of African Americans and Hispanics, the two subgroups of elders at highest risk for experiencing health disparities in the USA. ${ }^{13}$ We hypothesised that a 6-month lifestyle intervention leads to reduced decline in physical health, mental wellbeing and cognitive functioning among ethnically diverse older people across heterogeneous service delivery contexts and achieves these results in a cost-effective manner.

\section{METHODS}

A detailed description of methodological and logistical issues surrounding the wider Well Elderly 2 study is presented in a previous publication. ${ }^{14}$ 
Methodology for the randomised controlled trial study component is described below.

\section{Participants}

The research participants were 460 men and women aged 60-95 years. All participants were residents of, users of or visitors to the study recruitment sites; demonstrated no overt signs of psychosis or dementia (based on a cursory screening procedure) and were able to complete the study assessment battery (with assistance, if necessary). All prospective participants completed the informed consent process prior to study entry.

Participants were recruited from 21 sites in the greater Los Angeles area, including 9 senior activity centres, 11 senior housing residences and 1 graduated care retirement community. Recruitment strategies included providing sign-up booths, giving presentations at meetings and social events and distributing flyers and posters.

Recruitment was undertaken in two successive cohorts to reduce temporal influences on study outcomes, overcome logistical difficulties, minimise interactions among participants and allow adjustments in ethnic stratification. Individuals in cohort $1(n=205)$ entered the study between November 2004 and June 2005, whereas those in cohort $2(n=255)$ entered the study between March and August 2006.

The study protocol was approved by the Institutional Review Board at the University of Southern California prior to participant recruitment and conformed to the principles of the Declaration of Helsinki. Furthermore, the study was monitored by a five-member data safety monitoring board.

\section{Study design}

Participants were randomly assigned to the intervention or notreatment control condition for their first 6 months of study involvement. All participants were baseline-tested before starting and post-tested after completing their respective conditions, with outcome variables consisting of perceived health, mental (psychosocial) well-being and cognition measures. To promote experimental control, each site served as a separate research unit within which condition assignments were made. On completion of baseline testing, each participant was assigned to condition by the project managers based on a computer-generated random number sequence.

Reflecting a crossover design component, control group participants undertook the intervention during the 6-month period immediately following the trial's main experimental phase. This strategy enabled secondary analyses of pre-to-post intervention-based change involving control participants and was consistent with ethical treatment of human participants.

The Well Elderly 2 study did not include a social control treatment arm. This omission, which reduced non-essential costs and logistical challenges, was justified based on our previous study's finding of no difference between a social activity control group and a no-treatment control group. ${ }^{6}$

\section{Treatment}

The intervention largely followed the one manualised in the original Well Elderly study. ${ }^{6}{ }^{15}$ Key aspects of the intervention, including its crucial elements and modular content areas, are detailed in table 1 . The intervention consisted of small group and individual sessions led by a licensed occupational therapist. Typically, each group had six to eight members, all recruited from the same site and treated by the same intervener. Monthly community outings were scheduled to facilitate direct experience with intervention content such as the use of public transportation. Due to the overt nature of lifestyle programmes, neither the therapists nor the treated participants were blind to the intervention. However, the

Table 1 The Well Elderly Lifestyle Redesign $₫$ intervention

\begin{tabular}{|c|c|}
\hline Objective & To assist elders in developing a personally meaningful, healthy lifestyle that is sustainable within the fabric of their everyday routines \\
\hline Responsible professionals & $\begin{array}{l}\text { Occupational therapists licensed to practice in California } \\
\text { Therapists completed } 40 \mathrm{~h} \text { of training to standardise provision of the Lifestyle Redesign }{ }^{\circledR} \text { protocol in accord with manualised } \\
\text { specifications } \\
\text { Therapists participated in weekly or bi-weekly meetings with the on-site project director and manager to ensure intervention } \\
\text { fidelity and quality control }\end{array}$ \\
\hline Format & $\begin{array}{l}\text { Weekly 2-h small group sessions led by a licensed occupational therapist } \\
\text { - Didactic presentation; peer exchange; direct experience (participation in activities); personal exploration (application of content } \\
\text { to self) }\end{array}$ \\
\hline Key elements of intervention & $\begin{array}{l}\text { Identification and implementation of feasible and sustainable activity-relevant changes } \\
\text { Development of plans to overcome mundane obstacles to enacting activity-relevant changes (eg, bodily aches or transportation } \\
\text { limitations) }\end{array}$ \\
\hline Modular content areas & $\begin{array}{l}\text { Impact of everyday activity on health } \\
\text { - How activity contributes to health; impact of ageing on activity patterns; incorporating physical and mental exercise into everyday life } \\
\text { Time use and energy conservation } \\
\text { - Evaluating time use; assessing daily energy patterns; energy conservation and joint protection; adapting activities and daily routines } \\
\text { Transportation utilisation } \\
\text { - Available forms of transportation; cost, convenience, and accessibility; impact of transportation resources/constraints on activity; } \\
\text { transportation as activity or facilitator of activity } \\
\text { - Home and community safety } \\
\text { - Review common safety hazards; fall prevention; home safety evaluation; balancing safety concerns with activity; preparing for } \\
\text { emergencies; provision of assistive devices and home modifications as needed } \\
\text { Social relationships } \\
\text { - Building social connections through activity; impact of ageing on social relationships; obstacles to effective communication; coping } \\
\text { with loss and grief } \\
\text { Cultural awareness } \\
\text { - How culture influences everyday activity; strategies for managing cultural differences in everyday life; learning about different cultures } \\
\text { Goal setting } \\
\text { - Developing attainable goals for health improvements through daily activity } \\
\text { Changing routines and habits } \\
\text { - Evaluating temporal rhythms of activity (daily, weekly, monthly, annual); assessing readiness to change; implementing sustainable } \\
\text { changes to activity patterns }\end{array}$ \\
\hline
\end{tabular}


interveners and participants were blind to the study design and hypotheses.

\section{Assessment}

Testing sessions typically occurred in groups of 4-29 elders and took place in recreation or meeting rooms at the study sites. Assessment of health-related quality of life, life satisfaction and depression was based on self-rated questionnaires and was overseen by trained testers who were blind to the participants' condition assignments. Spanish versions of the questionnaires were provided for individuals in a Spanish study segment $(n=67)$. The cognitive tests were conducted individually, in a private area adjacent to the main testing room, at varying points during the testing session.

Norm-based scores on Version 2 of the 36-Item Short-Form Health Survey (SF-36v2) were used to measure perceived physical health and aspects of mental well-being. ${ }^{16}$ The SF-36 is appropriate for use with older populations ${ }^{17}$ as well as ethnically diverse samples ${ }^{18}$ and is consistent with more objective health measures. ${ }^{19}$

The Center for Epidemiologic Studies Depression (CES-D) Scale ${ }^{20}$ was used to assess depressive symptoms. The CES-D is sensitive to change in depressive status over time and has been successfully used to assess ethnically diverse older people. ${ }^{21} 22$

Life satisfaction was measured by the Life Satisfaction IndexZ (LSI-Z), a 13-item measure developed specifically for older people. $^{23}$ The LSI-Z is internally consistent, possesses criterionrelated validity and was sensitive to the effects of lifestyle intervention in our previous trial. ${ }^{6} 23$

Three cognitive outcome variables, immediate recall, delayed recall and recognition, were measured by the word list procedure developed by the Consortium to Establish a Registry of Alzheimer's Disease. ${ }^{24}$ Reliability and validity of the Consortium to Establish a Registry of Alzheimer's Disease Word List Memory task has been established for older people with and without dementia. ${ }^{25}$ Selective attention was measured by median reaction time on a widely used computer-based visual search task, ${ }^{26}$ with lower scores indicating higher cognitive functioning. Previous research has shown that this procedure is sensitive to individual differences in selective attention. ${ }^{26} \mathrm{~A}$ final cognitive outcome variable, psychomotor speed, was assessed by the Digit Symbol Substitution Task of the Weschler Adult Intelligence Scale-Revised, ${ }^{27}$ which is associated with general cognitive ability and physical health. ${ }^{28}$

\section{Statistical analyses}

\section{Data quality control}

All data entry, data management and statistical analyses were performed by the project's data analysis centre. Summary scores on each assessment were calculated using instrument-specific algorithms. Standard procedures were used to impute missing responses.

\section{Intent-to-treat analysis}

Power calculations were based on the assumption of an effect size of 0.32 (the average value for significant outcomes in our previous trial), a $10 \%$ attrition rate, use of a one-tailed test for adjusted change score differences and a $0.05 \alpha$ level. For the initially planned sample size of 220 per group, power to detect a between-group difference was estimated to be $93 \%$.

Student $t$ tests or $\chi^{2}$ tests were used to assess (1) the equivalence of the intervention and control groups on demographic and outcome variables at baseline, (2) the differences among participants who were evaluable versus non-evaluable at the time of post-testing and (3) the association of treatment assignment with evaluable versus non-evaluable status.

To test for intervention effects, for each of the 17 outcome variables signed change scores (post-test minus baseline) were generated, and analysis of covariance was performed to determine whether change scores of the intervention group were more favourable than those of the control group. In these analyses, the study cohort and the baseline score on the outcome variable were used as covariates, along with demographic or cognitive variables significantly related to the total sample's baseline to post-test change scores.

One-tailed statistical tests, conducted at the $0.05 \alpha$ level, were used to assess the hypotheses of positive treatment effects. Onesided tests were used because (1) the intervention could be justifiably linked to an expectation of positive health outcomes based on existing theory ${ }^{29},(2)$ a positive experimental effect had previously been demonstrated in the first Well Elderly trial ${ }^{6-8}$ and (3) the directional hypotheses were prespecified prior to the start of the study.

\section{Secondary analyses}

In a secondary non-experimental analysis of the intervention's effects, pre-to-post change was examined for individuals who were initially assigned to the control group, but received the intervention during the second 6 months of their study participation. The 17 outcome variable mean change scores were each separately tested through analysis of a covariance using a mixedeffects model with repeated measurements. The covariates were those included in the primary intent-to-treat comparison. For each outcome variable, a one-sided significance test was conducted at the $0.05 \alpha$ level.

Cost-effectiveness was evaluated by applying a cost per quality-adjusted life year (cost per OALY) methodology. ${ }^{8}$ SF-36v2 change scores were used to calculate differences in utility scores for the intervention and control groups. Intervention costs for the treatment group were calculated on an intent-to-treat basis and were based on an annual full-time equivalent salary of $\$ 62400$ (plus 32\% fringe benefits) for the treating therapists as reported by the Bureau of Labour Statistics. ${ }^{30}$ These costs were translated into UK costs using an hourly occupational therapist wage rate of $£ 24 .{ }^{31}$

Descriptive statistics were calculated to document the extent of treatment adherence (percentage of sessions attended) among all individuals who were in the study at the outset of their assigned intervention period within either the initial experimental or crossover phase.

\section{RESULTS}

\section{Description of study cohort}

Figure 1 presents the CONSORT diagram for the study. Of 838 study eligible individuals, 460 enrolled in the study. Of the 460 participants, 232 were randomly assigned to the intervention group and 228 to the control group. A total of 360 participants completed post-testing.

Table 2 presents baseline characteristics for all participants combined and by treatment arm. No significant differences were found between the intervention and control groups at baseline.

Relative to individuals who were non-evaluable, evaluable participants were more often engaged in productive activities such as volunteering, caregiving or part time work $(p<0.01)$ and had higher baseline scores on delayed recall and psychomotor speed (ps<0.05). Evaluable versus non-evaluable status was not associated with condition assignment (81\% of intervention versus $76 \%$ of control participants were evaluable, $\mathrm{p}>0.05)$. 


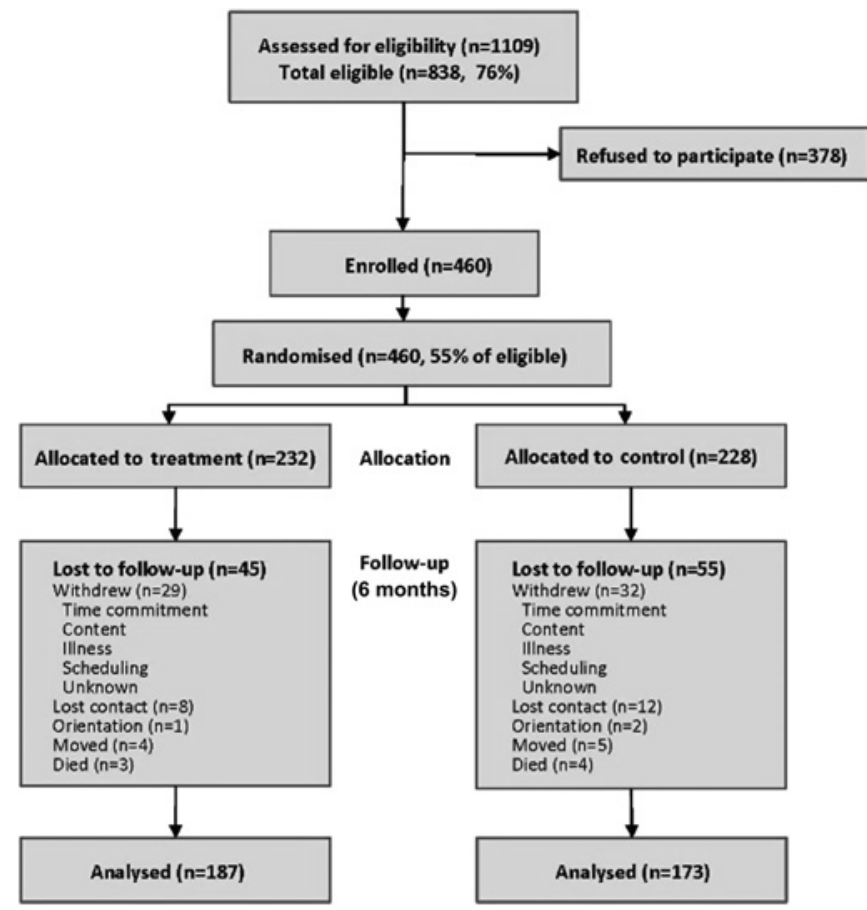

Figure 1 CONSORT diagram.

\section{Intent-to-treat analysis}

Table 3 summarises the results of the intent-to-treat analysis for evaluable participants. Analyses of covariance revealed a significant benefit due to the lifestyle intervention for five SF-36v2 outcomes: bodily pain $(p<0.02)$, vitality $(p<0.03)$, social function $(p<0.04)$, mental health $(p<0.03)$ and mental composite $(p<0.03)$. Physical function and physical composite were marginally significant $(p<0.10)$. In addition, a positive intervention effect was found for the LSI-Z $(p<0.03)$ and CES-D Scale $(\mathrm{p}<0.03)$.

A total of 405 individuals were enrolled in the study at the time they were expected to begin participating in the intervention. On average, participants attended $56 \%$ of the scheduled sessions. Sixty-nine $(17 \%)$ individuals did not attend any intervention sessions. Among participants who attended more than one session, the overall attendance rate was $70 \%$.

Among participants who received the intervention, six studyrelated adverse events were reported in the first 6-month experimental phase: two falls, three minor lacerations and one instance of transient dissatisfaction with the intervention. During the crossover period, in which the former control group received the intervention, four study-related adverse events were reported: two interpersonal conflicts, one partial fall and one instance of back pain following a session. No serious adverse events were reported.

\section{Secondary analyses}

Table 4 summarises the results of the change score analysis for the 137 participants who received the intervention during the second 6 months of their study involvement. For mental and physical well-being, all outcomes reflected positive change and seven outcomes were significant $(p s<0.05)$. Among the cognitive variables, significant pre-to-post improvement was found for immediate recall $(p=0.05)$, delayed recall $(p<0.0001)$, recognition $(p<0.01)$ and psychomotor speed $(p<0.01)$.

The intervention costs averaged $\$ 783$ per participant. Converting to UK wages and prices, the intervention costs averaged $£ 472.5$. The treatment group, relative to the control group, had an average OALY increment of $0.038(p<0.02)$. The base case cost per OALY was estimated at $\$ 41218$. Converting to UK wages and prices the base case cost per OALY was $£ 24868$, a number within the range that is often considered cost-effective by the UK National Institute of Health and Clinical Excellence.

\section{DISCUSSION}

Overall, this trial demonstrated that a 6-month preventive lifestyle-oriented intervention has positive effects for a sample of ethnically diverse older people recruited from a variety of community sites. Statistically significant results were found for multiple mental well-being variables, including vitality, social function, mental health, the mental health composite index, life satisfaction and depressive symptomatology. In the case of self-rated physical health, the intervention reduced perceptions of bodily pain and led to marginally significant improvements on the physical health composite and physical function scales. In general, however, the intervention had a larger impact for mental, as opposed to physical, health and well-being.

The basic pattern of positive experimental outcomes was mirrored among individuals who received the intervention during the crossover study phase. Furthermore, the intervention's salutary effect was consistent across two different time periods, as evidenced by the lack of an interaction between the study cohort variable and experimental treatment condition in affecting outcomes ( $p>0.10$ for all 17 dependent variables).

Because elders who are willing to undertake a research-based lifestyle intervention would potentially enrol in such an intervention in naturalistic non-research contexts, the study sample arguably approximates the pool of intervention participants in real-life applications. Based on information in figure 1 and table 2 , approximately one-half of the population of ethnically diverse community-dwelling older people who live in senior housing or frequent activity centres could be expected to participate in the intervention. This participation rate, in connection with the correlation of evaluability with productive activity and selected indices of cognitive functioning, suggests that the intervention may generalise most strongly to elders who are relatively capable or motivated.

As reported in table 3, no intervention effect was found for the cognitive outcomes. Although significant gains in immediate recall, delayed recall, recognition and psychomotor speed were present for the former control participants during the time they received the intervention (table 4), similar gains were also observed 6 months after completion of the intervention in the previous experimental group, suggesting a possible practice effect. Thus, convincing evidence for an intervention effect on cognition is lacking. At least two reasons may underlie this null result. First, it is possible that the 6-month experimental duration of the study was insufficient for cognitive changes to emerge. Second, due to the intervention's relative lack of direct content pertaining to cognition, such outcomes may have been less strongly linked to the intervention than mental well-being and physical outcomes.

\section{Strengths and limitations in relation to other studies}

Previously, a limited number of randomised trials, apart from the two Well Elderly studies, have been conducted to examine the effects of lifestyle-based interventions on mental well-being or physical outcomes of older people living in community or assisted living settings. ${ }^{6-11}$ However, the study samples have 
Table 2 Demographic and outcome variables at baseline of sample stratified by treatment $(n=460)$

\begin{tabular}{|c|c|c|c|c|}
\hline Demographic variables & Treatment $(n=232)$ & Control $(n=228)$ & Total $(n=460)$ & p Value* (two-sided) \\
\hline \multicolumn{5}{|l|}{ Sex, n (\%) } \\
\hline Male & $70(30.2)$ & $87(38.2)$ & $157(34.1)$ & 0.07 \\
\hline Female & $162(69.8)$ & $141(61.8)$ & $303(65.9)$ & \\
\hline $60-64, \mathrm{n}(\%)$ & $24(10.3)$ & $23(10.1)$ & $47(10.2)$ & 0.50 \\
\hline $65-69, \mathrm{n}(\%)$ & $40(17.2)$ & $39(17.1)$ & 79 (17.2) & \\
\hline $70-74, \mathrm{n}(\%)$ & $50(21.6)$ & $44(19.3)$ & $94(20.4)$ & \\
\hline $85+, \mathrm{n}(\%)$ & $22(9.5)$ & $25(11.0)$ & $47(10.2)$ & \\
\hline \multicolumn{5}{|l|}{ Race, $n(\%)$} \\
\hline White & $85(36.6)$ & $87(38.2)$ & $172(37.4)$ & 0.44 \\
\hline Black/African American & $78(33.6)$ & $71(31.1)$ & $149(32.4)$ & \\
\hline Hispanic or Latino & $49(21.1)$ & $43(18.9)$ & $92(20.0)$ & \\
\hline Some college or technical school & 77 (33.2) & $81(35.5)$ & $158(34.4)$ & \\
\hline Four years of college or more & $38(16.4)$ & $39(17.1)$ & 77 (16.7) & \\
\hline \multicolumn{5}{|l|}{ Annual income $\neq, \mathrm{n}(\%)$} \\
\hline$\$ 0 \sim \$ 11999$ & $123(53.7)$ & $117(53.2)$ & $240(53.5)$ & 0.77 \\
\hline$\$ 12000-\$ 23999$ & $51(22.3)$ & $56(25.5)$ & $107(23.8)$ & \\
\hline$\$ 24000-\$ 35999$ & $25(10.9)$ & $24(10.9)$ & $49(10.9)$ & \\
\hline$\$ 36000+$ & $30(13.1)$ & $23(10.4)$ & $53(11.8)$ & \\
\hline \multicolumn{5}{|l|}{ Income support§, n (\%) } \\
\hline One person & $194(84.4)$ & $178(80.2)$ & $372(82.3)$ & 0.25 \\
\hline More than one person & $36(15.6)$ & $44(19.8)$ & $80(17.7)$ & \\
\hline \multicolumn{5}{|l|}{ Retirement status, n (\%) } \\
\hline Senior centre: multipurpose & $71(30.6)$ & $74(32.5)$ & $145(31.5)$ & \\
\hline Senior centre: parks and recreation & $37(16.0)$ & $37(16.2)$ & $74(16.1)$ & \\
\hline \multicolumn{5}{|l|}{ Neighbourhood score $†$ T } \\
\hline Neighbourhood score (1-7) & $4.6(1.4)$ & $4.6(1.4)$ & $4.6(1.4)$ & 0.93 \\
\hline \multicolumn{5}{|l|}{ Outcome variables $\dagger$} \\
\hline \multicolumn{5}{|l|}{ SF-36 V. 2} \\
\hline Bodily pain & $43.24(11.48)$ & $44.47(11.56)$ & $43.85(11.52)$ & 0.25 \\
\hline Physical function & $38.65(11.98)$ & $38.38(12.33)$ & $38.51(12.14)$ & 0.81 \\
\hline Role physical & $40.47(11.60)$ & $41.60(10.07)$ & $41.03(10.87)$ & 0.26 \\
\hline General health & $44.66(10.15)$ & $44.88(10.46)$ & $44.77(10.29)$ & 0.82 \\
\hline Mental health & 47.35 (11.79) & $47.59(11.32)$ & $47.47(11.54)$ & 0.83 \\
\hline Role emotional & $39.04(14.04)$ & $40.76(12.39)$ & $39.89(13.26)$ & 0.17 \\
\hline Social functioning & $44.23(11.71)$ & $45.82(10.61)$ & $45.02(11.19)$ & 0.13 \\
\hline Vitality & $49.86(9.82)$ & $50.14(9.75)$ & $50.00(9.78)$ & 0.76 \\
\hline Physical composite & $41.09(10.06)$ & $41.43(10.60)$ & $41.26(10.32)$ & 0.72 \\
\hline Mental composite & $46.90(11.74)$ & $48.05(10.81)$ & 47.47 (11.29) & 0.27 \\
\hline \multicolumn{5}{|l|}{ Life satisfaction-Z } \\
\hline $16.94(5.56)$ & $16.76(5.72)$ & $16.85(5.64)$ & 0.73 & \\
\hline \multicolumn{5}{|l|}{ CES-D Scale } \\
\hline $14.32(10.89)$ & $13.13(10.91)$ & $13.73(10.91)$ & 0.24 & \\
\hline \multicolumn{5}{|l|}{ CERAD-memory } \\
\hline Immediate recall & $4.07(1.71)$ & $4.09(1.48)$ & $4.08(1.60)$ & 0.87 \\
\hline Delayed recall & $5.02(2.22)$ & $4.78(2.23)$ & $4.90(2.23)$ & 0.25 \\
\hline
\end{tabular}


Table 2 Continued

\begin{tabular}{|c|c|c|c|c|}
\hline Demographic variables & Treatment $(n=232)$ & Control $(n=228)$ & Total $(n=460)$ & p Value* (two-sided) \\
\hline \multicolumn{5}{|l|}{ Visual search } \\
\hline Median reaction time & $1413.59(647.40)$ & $1355.41(596.80)$ & $1385(623.40)$ & 0.34 \\
\hline \multicolumn{5}{|l|}{ Psychomotor speed } \\
\hline
\end{tabular}

Age is calculated from date of birth and baseline evaluation date; in the category race, 'Other' includes Native Hawaiian or Other Pacific Islander, American Indian/Alaska Native, Multiracial other and refused.

${ }^{*} \chi^{2}$ tests were performed for categorical variables, and Student $t$ tests were performed for continuous variables.

†Mean (SD).

fIncome: 11 refused ( 3 in the treatment group and 8 in the control group).

SIncome support: 8 refused ( 2 in the treatment group and 6 in the control group).

IBased on project managers' ratings of the overall quality of the neighbourhood in which the site was located.

CES-D Scale, Center for Epidemiologic Studies Depression Scale; CERAD, Consortium to Establish a Registry of Alzheimer's Disease; SF-36 V. 2, Version 2 of the 36-Item Short-Form Health

Survey.

been largely limited to white older people, the interventions have lacked an individually tailored comprehensive focus and the cost-effectiveness analyses were not performed. The Well Elderly 2 study is the first investigation that addressed these concerns while documenting the effectiveness of a lifestyle intervention under widely varying naturalistic circumstances.

The effect size estimates for significant outcomes in the primary intent-to-treat analysis ranged from 0.14 to 0.23

Table 3 Intent-to-treat analysis of outcome change scores (post-baseline) for evaluable participants stratified by intervention group ( $\mathrm{n}=360$ )

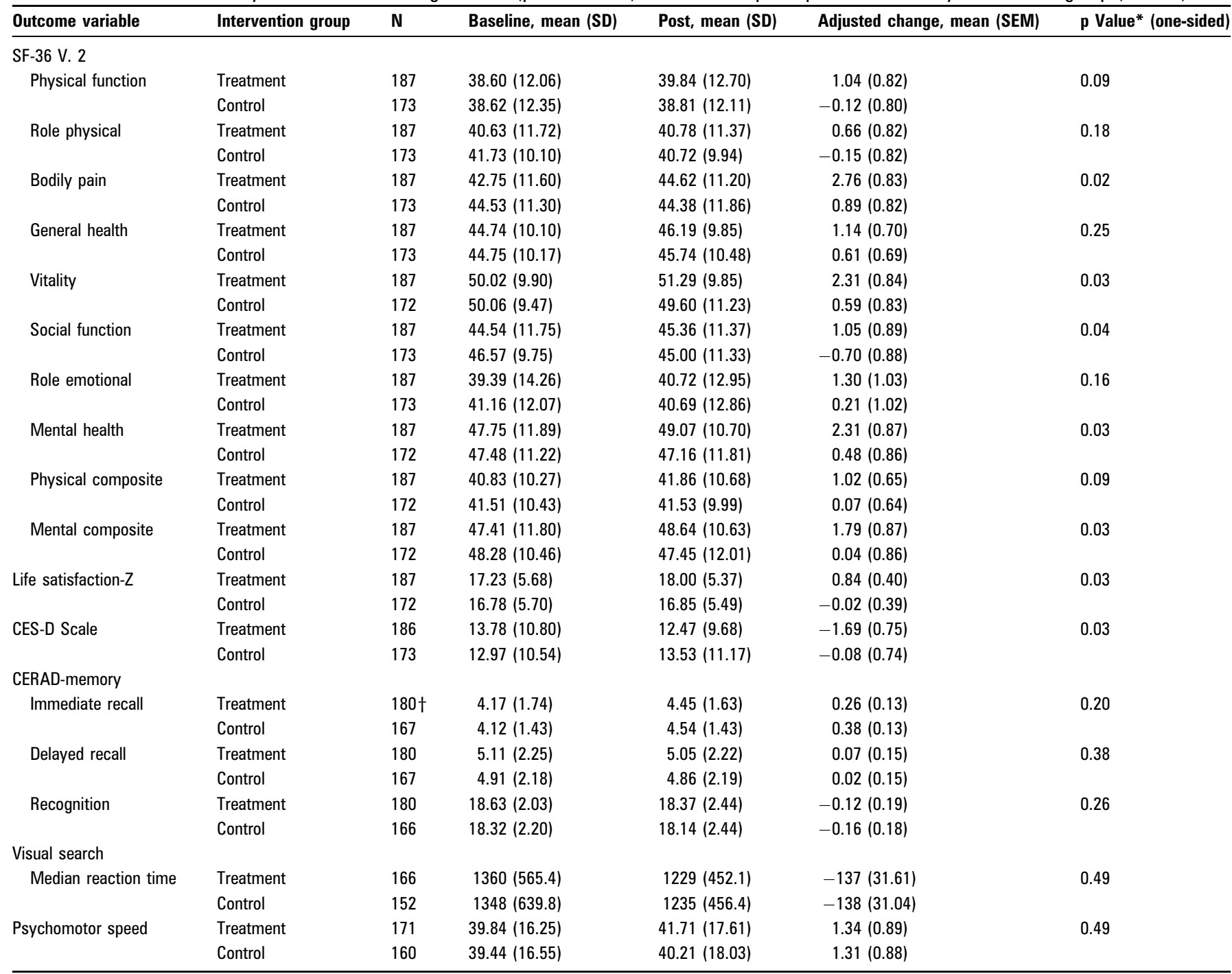

Covariates were (1) baseline value, (2) cohort (1, 2), (3) age (continuous), (4) sex (male, female), (5) race (white, black, Hispanic, Other), (6) education (<high school, high school, some college and college plus), (7) site (graduated care retirement community, senior residence, senior centre-multipurpose, senior centre-parks and recreation), (8) neighbourhood score (1-7), (9) CERAD-recognition, (10) psychomotor speed.

*p Values were obtained using analysis of covariance.

tReasons for not completing cognitive testing: visual or other physical impairment or scheduling conflicts.

CES-D Scale, Center for Epidemiologic Studies Depression Scale; CERAD, Consortium to Establish a Registry of Alzheimer's Disease; SF-36 V. 2, Version 2 of the 36-Item Short-Form Health Survey. 
Table 4 Analysis of covariate-adjusted outcome change scores for control group participants who crossed over to the intervention ( $n=137$ )

\begin{tabular}{|c|c|c|c|c|c|}
\hline Outcome variable & $\mathbf{N}$ & Pre, mean (SD) & Post, mean (SD) & Adjusted change, mean (SEM) & p value* (one-sided) \\
\hline \multicolumn{6}{|l|}{ SF-36 V. 2} \\
\hline Physical function & 137 & $38.90(12.09)$ & $40.08(12.04)$ & $1.18(0.81)$ & 0.07 \\
\hline Role physical & 137 & $40.46(10.34)$ & $42.30(9.81)$ & $1.84(0.95)$ & 0.03 \\
\hline General health & 137 & $45.77(10.09)$ & $46.03(9.24)$ & $0.27(0.68)$ & 0.34 \\
\hline Vitality & 136 & 50.19 (11.32) & $51.77(9.00)$ & $1.53(0.78)$ & 0.03 \\
\hline Social function & 137 & $45.18(11.44)$ & $46.18(9.74)$ & $1.00(0.94)$ & 0.15 \\
\hline Physical composite & 136 & $41.45(9.95)$ & $42.49(9.81)$ & $0.69(0.64)$ & 0.07 \\
\hline Mental composite & 136 & $47.74(12.26)$ & $49.42(10.56)$ & $1.63(0.91)$ & 0.04 \\
\hline Life satisfaction-Z & 136 & $16.65(5.51)$ & $17.44(5.17)$ & $0.79(0.37)$ & 0.02 \\
\hline CES-D Scale & 137 & $13.39(11.05)$ & $11.78(9.21)$ & $-1.66(0.76)$ & 0.01 \\
\hline \multicolumn{6}{|l|}{ CERAD-memory } \\
\hline Immediate recall & $127 \dagger$ & $4.60(1.45)$ & $4.87(1.71)$ & $0.24(0.15)$ & 0.05 \\
\hline Psychomotor speed & 123 & $40.39(17.50)$ & 42.99 (17.82) & $2.19(0.96)$ & 0.01 \\
\hline
\end{tabular}

Covariates were (1) cohort (1, 2), (2) age (continuous), (3) sex (male, female), (4) race (white, black, Hispanic, Other), (5) education (<high school, high school, some college, college plus), (6) site (graduated care retirement community, senior residence, senior centre-multipurpose, senior centre - parks and recreation), (7) neighbourhood score (1-7), (8) CERAD-recognition (pretest), (9) psychomotor speed (pre-test).

*p Values were obtained using a mixed model with repeated measurement.

†Reasons for not completing cognitive testing: visual or other physical impairment or scheduling conflicts.

CES-D Scale, Center for Epidemiologic Studies Depression Scale; CERAD, Consortium to Establish a Registry of Alzheimer's Disease; SF-36 V. 2, Version 2 of the 36-Item Short-Form Health Survey.

(mean $=0.17)$. These values are smaller than those obtained in our previous trial, which on average exceeded 0.30. In interpreting this difference, it should be noted that the current investigation included design characteristics (eg, a more heterogeneous sample, the inclusion of relatively distal outcome measures, the enlistment of sites varying in their degree of administrative support and investment and a shortened intervention period) that are not conducive to yielding large effect sizes. $^{12}{ }^{32}$ By way of comparison, the effect magnitude approximates the level obtained in a large multisite trial of a lifestyle intervention for ethnically diverse care givers of relatives with dementia. $^{33}$

An additional consideration concerning the magnitude of the experimental effect is that the estimated base case cost per OALY of US \$41218/ UK $£ 24868$ is low enough for the intervention to qualify as cost-effective. ${ }^{34} 35$ Given this result, the intervention is viable as a treatment option in multi-ethnic community-based contexts.

\section{Unanswered questions and future research}

One unanswered question pertains to the absence of a readinessto-change screen. It has been recommended that controlled trials should exclude individuals who are unlikely to adhere to the study protocol or benefit from the intervention. ${ }^{32}$ Underscoring this notion, behavioural and health promotion studies reveal a strong relationship between subject readiness-to-change and protocol adherence and study outcomes. ${ }^{36}$ We believe that the study dropout rate might have been mitigated, or the experimental effect increased, if we had used readiness-to-change as a criterion for admissibility and used such scores to modify the intervention protocol.

Several potential future research extensions should be noted. First, although in the current study the intervention demonstrated excellent reach in extending to urban, communitydwelling older people from different age, gender, racial/ethnic and socioeconomic strata (see figure 1 and table 2), there is a need to test the benefits, as well as the cost-effectiveness, of lifestyle-oriented interventions when administered to groups with varying daily living circumstances such as those who reside in nursing homes or rural environments. Second, there is a need to document which aspects of complex multifaceted lifestyle interventions are most important in producing positive outcomes. Finally, in designing future lifestyle interventions for older people, inclusion of modular content that directly addresses cognitive functioning should be considered. In testing for such cognitive benefits, a follow-up period longer than that

\section{What is already known on this subject}

Older people are at increased risk for declines in mental wellbeing, physical functioning and loss of independence.

- Lifestyle interventions have been shown to reduce age-related declines under carefully controlled conditions.

- Full-scale trials of the effectiveness and cost-effectiveness of comprehensive lifestyle interventions on health outcomes administered to ethnically diverse elders in a variety of community settings are lacking.

\section{What this study adds}

- A lifestyle-oriented occupational therapy intervention promotes elder's mental well-being across community-based settings in a cost-effective manner.

- This intervention is widely applicable to those at high risk for poor health due to low socioeconomic status. 
used in the current study should be included insofar as such effects may develop slowly over time.

\section{CONCLUSIONS}

In conclusion, the Well Elderly 2 study documented the effectiveness of the intervention when (1) applied to a sample of older people at high risk for experiencing health disparities, (2) delivered within a 6-month time interval and (3) implemented in various community settings. The findings of this study are particularly relevant today when escalating healthcare costs are being attributed largely to the ageing population and related increases in depressive symptomatology, poor mental well-being and chronic illness. ${ }^{1}{ }^{37} 38$ As a consequence, healthcare policy specialists and governmental agencies are recognising that preventive and wellness care for older people must be a key element in healthcare provision. ${ }^{1}{ }^{39}$ The results of the Well Elderly 2 study demonstrate that because the intervention is cost-effective and applicable on a wide-scale basis, it has the potential to promote physical and mental well-being in this population.

\section{Author affiliations}

1 Division of Occupational Science and Occupational Therapy, School of Dentistry, University of Southern California, Los Angeles, California, USA

${ }^{2}$ Department of Preventive Medicine, Institute for Health Promotion and Disease Prevention Research, Keck School of Medicine, University of Southern California, Los Angeles, California, USA

${ }^{3}$ Department of Psychology, California State University, Fullerton, California, USA

${ }^{4}$ School of Social Work, University of Southern California, Los Angeles, California, USA

${ }^{5}$ Leonard Davis School of Gerontology, University of Southern California, Los Angeles, California, USA

${ }^{6}$ Center for Interdisciplinary Salivary Bioscience Research, The Johns Hopkins School of Nursing, Baltimore, Maryland, USA

${ }^{7}$ Department of Psychology, University of Southern California, Los Angeles, California, USA

${ }^{8}$ Family Medicine at Gabriel Park, Oregon Health and Science University, Portland, Oregon, USA

${ }^{9}$ Titus Family Department of Clinical Pharmacy and Pharmaceutical Economics and Policy, School of Pharmacy, University of Southern California, Los Angeles, California, USA

${ }^{10}$ Division of Biostatistics Statistical Consultation and Research Center, Department of Preventive Medicine, Keck School of Medicine, University of Southern California, Los Angeles, California, USA

Acknowledgements We thank program officer Sidney Stahl of the National Institute on Aging for his continuing support and helpful comments throughout the conduct of the project. The investigative team also expresses gratitude to the members of the data and safety monitoring board for their careful oversight and helpful suggestions: Sidney Stahl, NIA Behavioural and Social Research Program; Kenneth Ottenbacher, University of Texas-Medical Branch; Nancy Gibbs, Southern California Kaiser Permanente Medical Group; Thomas Belin, University of California at Los Angeles and Eleanor Durr of Altadena, CA. We also thank Carolyn Ervin, Peng Zhao and James Gardner for assisting in the early stages of data monitoring and analysis. Finally, we thank Stephanie Mielke and Janis Wise for their ongoing administrative support of the project, without whose diligent efforts this study could not have been initiated or completed.

Funding This research was supported by National Institutes of Health grant R01 AG021108 from the National Institute on Aging.

\section{Competing interests None.}

Ethics approval This study was conducted with the approval of the institutional review board at the University of Southern California, and all participants gave informed consent.

Contributors $\mathrm{FC}$, as principal investigator, obtained funding for the study and is guarantor. SPA, FC, JJ, MC, DM, JB, BW, C-PC, MJ-M, JH, BC, BGK and DAG provided substantial contributions to conception and design of the study. DM, JB, C-PC, MJ-M and BC acquired the data. SPA, MC, DM, C-PC, RW, MYL, CL, JH and DAG analysed the data. SPA, FC, JJ, MC, DM, JB, AM, C-PC, RW, MYL, CL, MJ-M, $\mathrm{JH}, \mathrm{BC}, \mathrm{BGK}$ and DAG interpreted the data. SPA, FC, JJ, MC, DM, AM, MYL, CL, JH and $\mathrm{BC}$ drafted the manuscript. All authors critically revised the manuscript for important intellectual content and approved the final version.

Provenance and peer review Not commissioned; externally peer reviewed.

\section{REFERENCES}

1. National Institute on Aging. Why Population Aging Matters: A Global Perspective Washington, DC: U.S. National Institutes of Health, 2007. http://www.nia.nih.gov/ Researchlnformation/ExtramuralPrograms/BehavioralAndSocialResearch/GlobalAging htm (accessed 7 Jul 2009).

2. Low G, Molzahn AE. Predictors of quality of life in old age: a cross-validation study. Res Nurs Health 2007;30:141-50.

3. Smith J, Borchelt M, Maier $\mathrm{H}$, et al. Health and well-being in the young old and oldest old. J Soc Issues 2002;58:715-32.

4. Rowe JW, Kahn RL. Successful aging. Aging (Milano) 1998;10:142-4.

5. Hendricks $\mathbf{J}$, Hatch LR. Theorizing lifestyle: exploring agency and structure in the life course. In: Bengston VL, Gans D, Putney N, et al, eds. Handbook of theories of aging. New York, NY: Springer Publishing Company, 2009:435-54

6. Clark F, Azen SP, Zemke R, et al. Occupational therapy for independent-living olde adults. A randomized controlled trial. JAMA 1997;278:1321-6.

7. Clark F, Azen SP, Carlson M, et al. Embedding health-promoting changes into the daily lives of independent-living older adults: long-term follow-up of occupational therapy intervention. J Gerontol B Psychol Sci Soc Sci 2001;56:P60-3.

8. Hay J, LaBree L, Luo $\mathrm{R}$, et al. Cost-effectiveness of preventive occupational therapy for independent-living older adults. J Am Geriatr Soc 2002;50:1381-8.

9. Peri K, Kerse N, Robinson E, et al. Does functionally based activity make a difference to health status and mobility? A randomised controlled trial in residential care facilities (The Promoting Independent Living Study; PILS). Age Ageing 2008:37:57-63.

10. Scott JC, Conner DA, Venohr I, et al. Effectiveness of a group outpatient visit mode for chronically ill older health maintenance organization members: a 2-year randomized trial of the cooperative health care clinic. J Am Geriatr Soc 2004:52:1463-70.

11. Wallace JI, Buchner DM, Grothaus L, et al. Implementation and effectiveness of a community-based health promotion program for older adults. J Gerontol A Biol Sci Med Sci 1998;53:M301-6.

12. Glasgow RE, Lichtenstein E, Marcus AC. Why don't we see more translation of health promotion research to practice? Rethinking the efficacy-to-effectiveness transition. Am J Public Health 2003;93:1261-7.

13. Smedley B, Stith A, Nelson A, eds. Unequal Treatment: Confronting Racial and Ethnic Disparities in Health Care. Washington, DC: Institute of Medicine 2003:29-79

14. Jackson J, Mandel D, Blanchard J, et al. Confronting challenges in intervention research with ethnically diverse older adults: the USC Well Elderly II Trial. Clin Trials 2009;6:90-101.

15. Mandel D, Jackson J, Zemke R, et al. Lifestyle Redesign: Implementing the Well Elderly Program. Bethesda, MD: AOTA Press, 1999:1-61.

16. Ware J, Kosinski M, Dewey J. How to Score Version 2 of the SF-36(r) Health Survey. Lincoln, Rl: QualityMetric Incorporated, 2000:23-102.

17. Hayes V, Morris J, Wolfe C, et al. The SF-36 health survey questionnaire: is it suitable for use with older adults? Age Ageing 1995;24:120-5.

18. Madsen F. Quality of life questionnaires for all respiratory diseases, every language and ethnic minorities. Are alternatives available? Respir Med 2000;94:187-9.

19. Brazier JE, Harper R, Jones NM, et al. Validating the SF-36 health survey questionnaire: new outcome measure for primary care. BMJ 1992;305:160-4.

20. Radloff L. The CES-D scale: a self report depression scale for research in the genera population. Appl Psych Meas 1977:1:385-401.

21. Foley K, Reed P, Mutran E, et al. Measurement adequacy of the CES-D among a sample of older African Americans. Psychiatr Res 2002;109:61-9.

22. Lewinsohn PM, Hoberman HM, Rosenbaum M. A prospective study of risk factors for unipolar depression. J Abnorm Psychol 1988;97:251-64.

23. Wood V, Wylie ML, Sheafor B. An analysis of a short self-report measure of life satisfaction: correlation with rater judgments. J Gerontol 1969:24:465-9.

24. Morris JC, Heyman A, Mohs RC, et al. The Consortium to Establish a Registry for Alzheimer's Disease (CERAD). Part I. Clinical and neuropsychological assessment of Alzheimer's disease. Neurology 1989;39:1159-65.

25. Welsh KA, Butters N, Mohs RC, et al. The Consortium to Establish a Registry for Alzheimer's Disease (CERAD). Part V. A normative study of the neuropsychological battery. Neurology 1994;44:609-14.

26. Lupien S, Lecours AR, Lussier I, et al. Basal cortisol levels and cognitive deficits in human aging. J Neurosci 1994;14:2893-903.

27. Weschler D. WAIS-R: Weschler Adult Intelligence Scale Revised. New York, NY: Psychological Corporation, 1981:1-20.

28. Lezak MD. Neuropsychological assessment. 3rd edn. New York, NY: Oxford University Press, 1995:376-9.

29. Jackson $\mathbf{J}$, Carlson $M$, Mandel $D$, et al. Occupation in lifestyle redesign: the well elderly study occupational therapy program. Am J Occup Ther 1998;52:326-36.

30. U.S. Bureau of Labor Statistics. Occupational Employment and Wages, May 2006. http://www.bls.gov/oes/2006/may/oes291122.htm (accessed 14 Jan 2009).

31. National Institute for Health and Clinical Excellence. Mental Well Being and Older People: Costing Report. London: 2008. http://guidance.nice.org.uk/PH16/ CostReport/pdf/English (accessed 14 Jan 2009).

32. Vamvakas EC. Rationale, objectives, and interpretation of randomized controlled trials. J Clin Apher 1997;12:130-9.

33. Gitlin LN, Belle SH, Burgio LD, et al. Effect of multicomponent interventions on caregiver burden and depression: the REACH multisite initiative at 6-month follow-up Psychol Aging 2003:18:361-74. 
34. Hay JW. Where's the value in health care? Value Health 2006;9:141-3.

35. McCabe C, Claxton K, Culyer AJ. The NICE cost-effectiveness threshold: what it is and what that means. Pharmacoeconomics 2008:26:733-44.

36. Prochaska J, Norcross J. Stages of change. Psychotherapy 2001;38:443-8.

37. Centers for Disease Control and Prevention. The State of Aging and Health in America 2007. U.S. Department of Health and Human Services, 2007 [updated 2008 June 30]. http://www.cdc.gov/aging/saha.htm laccessed 14 Jan 2009)

38. Allen J. Older People and Wellbeing. London Institute for Public Policy Research, 2008. http://www.ippr.org.uk (accessed 7 Jul 2009).

39. Baucus M. Call to Action: Health Reform 2009. U.S. Senate Finance Committee, 2008. http://finance.senate.gov/healthreform2009/home.html (accessed 14 Jan 2009).

\section{Journal of Epidemiology and Community Health alerts}

Sign up for our electronic table of contents alerts and you will never miss new issues of Journal of Epidemiology and Community Health when published online. Stay ahead and up to date by visiting jech.bmj.com. 


\section{Corrections}

Clark F, Jackson J, Carlson M, et al. Effectiveness of a lifestyle intervention in promoting the well-being of independently living older people: results of the Well Elderly 2 Randomised Controlled Trial. J Epidemiol Community Health 2012;66:9 782-790. The data in Table 2 were shifted to the left for the following outcome variables: Life satisfaction- $Z$, CES-D Scale, Psychomotor Speed. Each of those rows of data should be shifted one column to the right so that they match with the correct column headers.

J Epdemiol community Health 2012;66:1082. doi:10.1136/jech.2009.099754corr1 